\title{
Pathogens and symbionts in ticks: a survey on tick species distribution and presence of tick- transmitted micro-organisms in Sardinia, Italy
}

\author{
Correspondence \\ Giuseppe Satta \\ giuseppe.satta@izs-sardegna.it
}

Received 20 April 2010

Accepted 29 September 2010

\author{
Giuseppe Satta, Valentina Chisu, Pierangela Cabras, Francesco Fois \\ and Giovanna Masala
}

Istituto Zooprofilattico Sperimentale della Sardegna, Sassari, Italy

\begin{abstract}
A total of 1485 adult ticks were collected from mammalian hosts in south-eastern Sardinia, Italy, during the years 2007-2008. Ticks were identified and tested by PCR analysis for presence of Rickettsia species of the spotted fever group, Ehrlichia canis, Anaplasma phagocytophilum, Coxiella burnetii, Bartonella species and Leishmania species. Among all tick species examined (Rhipicephalus sanguineus, Rhipicephalus turanicus, Rhipicephalus bursa, Rhipicephalus pusillus, Hyalomma marginatum marginatum, Haemaphysalis sulcata and Dermacentor marginatus), only Hyalomma marginatum marginatum produced negative results. A total of 22 pools belonging to the three tick species Rhipicephalus sanguineus (0.9\%), Rhipicephalus turanicus (4.5\%) and Rhipicephalus pusillus (100\%) were positive for Rickettsia species, while a total of five pools belonging to Rhipicephalus sanguineus (0.09\%), Haemaphysalis sulcata $(16.7 \%)$ and D. marginatus $(7.8 \%)$ were positive for E. canis. Five pools of Rhipicephalus turanicus (1.8\%) were positive for A. phagocytophilum. Positivity for C. burnetii was found in seven pools belonging to three tick species: Rhipicephalus sanguineus (0.5\%), Rhipicephalus turanicus (0.3\%) and Haemaphysalis sulcata (4.4\%). Finally, four pools belonging to Rhipicephalus sanguineus (0.09\%), Rhipicephalus turanicus (0.7\%) and Rhipicephalus bursa (1.1\%) were positive for Bartonella species. Leishmania species DNA was not detected in any of the tick pools examined. Data presented here increase our knowledge on tick-borne diseases in Sardinia, and provide a useful contribution to understanding their epidemiology.
\end{abstract}

\section{INTRODUCTION}

Tick-borne diseases are an emerging medical and veterinary problem. Ticks are implicated in the transmission of different pathogens such as viruses, bacteria, protozoa and filarial nematodes (Dantas-Torres, 2008). In the last few decades, there has been an increasing interest in zoonotic tick-borne diseases, which are considered as one of the most important zoonoses in Europe (Parola \& Raoult, 2001). The family Ixodidae includes several relevant species, most of which belong to the genera Haemaphysalis, Rhipicephalus, Dermacentor, Amblyomma (DantasTorres, 2008) and Hyalomma (Black \& Piesman, 1994). Rhipicephalus bursa and Hyalomma marginatum marginatum occur in all bioclimatic zones, while Rhipicephalus turanicus and Rhipicephalus sanguineus occur essentially in the mesomediterranean bioclimatic zone. Dermacentor marginatus and Haemaphysalis sulcata are found frequently in the biotopes of the attenuated mesomediterranean and submediterranean bioclimates (Papadopoulos et al., 1996). Ixodid ticks can transfer members of the spotted fever group (SFG) rickettsiae to vertebrates via salivary secretions, and among themselves both transtadially and transovarially (Beninati et al., 2002). Canine monocytic ehrlichiosis is a cosmopolitan tick-borne disease of dogs that is primarily caused by Ehrlichia canis (Stich et al., 2002). Recently, Anaplasma phagocytophilum causing disease in humans and animals was also found in Ixodes ticks (Foley et al., 2008). Coxiella burnetii, the causative agent of Q fever 'worldwide zoonosis', has been detected in several tick species, but the role of ticks in transmitting the pathogen to humans is probably minimal (Psaroulaki et al., 2006). The potential role of ticks as vectors of Bartonella species has been only hypothesized (Angelakis et al., 2010). Ixodes ricinus ticks are competent vectors for Bartonella henselae, but further investigations are needed in order to evaluate their ability to transmit this pathogen (Cotté et al., 2008). Rhipicephalus sanguineus has been demonstrated to be susceptible to infection by Leishmania, and to be able to mediate its transmission to experimental hosts (Coutinho et al., 2005). Rhipicephalus sanguineus is considered a globalized tick and is able to transmit pathogens such as Rickettsia rickettsii (Dantas-Torres, 2008), Ehrlichia species and Anaplasma species (Sarih et al., 2005), C. burnetii 
(Bernasconi et al., 2002) and Leishmania infantum (Coutinho et al., 2005).

\section{METHODS}

Study area. Sardinia is the second largest island in the Mediterranean Sea, with an area of $23821 \mathrm{~km}^{2}$. Ogliastra, one of the two collection areas, is a region of great naturalistic importance located in southeastern Sardinia. On the northern side, Ogliastra is surrounded by the Gennargentu mountains, forests and green valleys covered by the typical Mediterranean maquis with Cistus, lentisk, myrtle and rosemary shrubs. The landscape is also characterized by cultivated coastal plains, watercourses and rocky sheer coasts. Many areas are dedicated to rearing and grazing of sheep, goats, bovines, swine and horses. Ogliastra is also an extraordinary habitat for wild animals such as mouflons, Sardinian deer, wild pigs and foxes and many birds. The second collection area, in the province of Cagliari, is located in southern Sardinia and is characterized by a wide diversity in geology, vegetation and landscape features, marked by the presence of mountains, great woods and Mediterranean maquis where rare animals such as wild cats, deer and wild pigs and several rare birds live. The rest is dedicated to rearing and grazing of farm animals.

Sample collection and identification. A total of 1485 ticks were collected from March to December, with peaks in May-June, during the years 2007-2008, from 80 dogs, 3 wild boars (Sus scrofa meridionalis), 36 sheep, 41 goats, 7 horses, 13 cattle, 1 deer (Cervus elaphus corsicanus) and 2 hedgehogs (Erinaceus europaeus italicus). Resident wild animals sampled during the study (deer, hedgehogs, wild boar) had been brought dead to our laboratories for necropsy analyses and tick capture. The other animals (dogs, sheep, goats, cattle and horses) from which ticks were removed came from farms, and the owners had provided the ticks using containment material. Ticks were removed from their host with tweezers and placed in vials with $70 \%$ ethanol at room temperature. Identification was carried out by observation with a binocular microscope $(\times 10-50)$, and ticks were classified into family, genus and species using the taxonomic keys and morphometric tables available for tick identification (Manilla, 1998).

DNA extraction and PCR assay. All adult ticks belonging to the same species and collected from the same animal were segregated into pools of five ticks each. The pooled samples were immersed in distilled water for $10 \mathrm{~min}$, dried on sterile filter paper, and crushed with a sterile scalpel in Eppendorf tubes. DNA extraction was performed using a DNeasy Blood \& Tissue kit (Qiagen) according to the manufacturer's instructions.

PCR was performed to evaluate the presence of Rickettsia species, E. canis, A. phagocytophilum, C. burnetii, Bartonella species and Leishmania species DNA in each pool using the GeneAmp PCR System 9700 (Applied Biosystems). The assay amplifies specifically a $500 \mathrm{bp}$ fragment of the $\operatorname{ompB}$ gene (F: 5'-CTAGTGCAGATGCAAATG-3'; R: 5'-GTTTGAAATGATAATTG-3') of Rickettsia SFG (Noda et al., 1997), 200 bp of the $p 30$ gene (F: $5^{\prime}$-CATGATTGGGATGGAAGTCCAATAC-3'; R: 5'-ATGGCTGCCGATGTGTGATG-3') of $E$. canis (Stich et al., 2002), 293 bp of the $16 \mathrm{~S}$ rRNA gene (F: 5'TGTAGGCGGTTCGGTAAGTTAAAG-3'; R: 5'-CTTAACGCGTTAGCTACAACACAG-3') of A. phagocytophilum (Kolbert, 1996), 257 bp of the superoxide dismutase gene (F: 5'-ACTCAACGCACTGGAACCGC-3'; R: 5'-TAGCTGAAGCCAATTCGCC-3') of C. burnetii (Stein \& Raoult, 1992), 298 bp of the 16S rRNA gene (F: 5'-GAGATGGCTTTTGGAGATTA-3'; R: 5' -CCTCCTTCAGTTAGGCTGGG-3') of Bartonella species (Sander et al., 1999) and 358 bp of the SSU rRNA gene (F: 5'-TCCCATCGCAACTTCGGT-3'; R: 5'-AAAGCGGGCGCGGTGCTG-3') of Leishmania species (van Eys et al., 1992).
Positive control DNAs were extracted from C. burnetii (Nine mile/I/ EP1), E. canis (ATCC-CRL-10390), B. henselae (Houston 1 ATCC 49882), Rickettsia conorii (SIMKO EP7) and L. infantum (MON 1). Human promyelocytic leukaemia (HL60) cells infected with A. phagocytophilum were used as a positive control. Water samples were included in all amplifications as a negative control. PCR products were resolved on a $1-1.5 \%$ agarose gel in $1 \times$ TAE buffer $(0.04 \mathrm{M}$ Tris/acetate, $0.001 \mathrm{M}$ EDTA). After electrophoresis at $100 \mathrm{~V}$ for $60 \mathrm{~min}$, gels were stained with ethidium bromide and examined over UV light.

Data analysis. In order to verify the repeatability of our results, PCR analysis was performed three times on each tick pool. Infection rate in tick pools was estimated using the formula maximum-likelihood estimation $(\mathrm{MLE})=1-(1-Y / X)^{1 / m}$ as described by Walter et al. (1980), where $Y=$ number of positive pools, $X=$ number of pools and $m=$ number of organisms per pool. This formula assumes that when a PCR product is positive from a pool of five ticks, only one tick in the pool is considered to be infected.

\section{RESULTS}

\section{Tick species and host distribution}

Seven species of ticks belonging to the order Ixodidae were identified among a total of 1485 adult ticks randomly collected from mammals in Sardinia: Rhipicephalus sanguineus, Rhipicephalus turanicus, Rhipicephalus bursa, Rhipicephalus pusillus, Hyalomma marginatum marginatum, Haemaphysalis sulcata and D. marginatus. The number of ticks collected from animals and used in this study were as follows: 970 from dogs; 180 from sheep; 205 from goats; 65 from cattle; 35 from horses; 10 from hedgehogs; 5 from deer; and 15 from wild boars. The percentages of tick species abundance in Sardinia are reported in Table 1.

A total of $92.3 \%$ of Rhipicephalus sanguineus tick species were removed from dogs. Rhipicephalus turanicus ticks were removed mostly from sheep and goats $(32.7 \%$ and $51.7 \%$, respectively). Rhipicephalus bursa specimens occurred mostly on sheep (38.9\%), but were also collected from a wide range of other hosts: $33.3 \%$ from horses; $16.7 \%$ from goats; $5.5 \%$ from cattle; and $5.5 \%$ from deer. Rhipicephalus pusillus ticks were found only in a hedgehog. Fifteen ticks identified as Hyalomma marginatum marginatum were removed from cattle. Haemaphysalis sulcata ticks were all detected in goats and sheep. Finally, ticks removed from wild boars were classified as $D$. marginatus $(1 \%)$. Tick association with mammal hosts is reported in Table 2.

\section{Detection of pathogens in ticks}

Six out of the seven tick species identified contained DNA of pathogens. Out of 209 pools of Rhipicephalus sanguineus, nine pools (eight from dogs and one from sheep) were positive for Rickettsia SFG; one pool from a dog was positive for E. canis, five pools (four from dogs and one from a goat) were positive for C. burnetii, and one pool from a dog was positive for Bartonella species. Twelve 
Table 1. Number of identified total tick pools, percentage of their estimated abundance with respect to the total number of ticks collected, pools testing positive for Rickettsia species, E. canis, A. phagocytophilum, C. burnetii and Bartonella species, and the rate of infection (RI) in pools from each tick species

\begin{tabular}{|c|c|c|c|c|c|c|c|}
\hline \multirow[t]{2}{*}{ Tick species } & \multirow[t]{2}{*}{ No. of pools } & \multirow{2}{*}{$\begin{array}{c}\text { Tick } \\
\text { abundance } \\
(\%)\end{array}$} & \multicolumn{5}{|c|}{ Positive pools } \\
\hline & & & Rickettsia (SFG) & E. canis & A. phagocytophilum & C. burnetii & Bartonella spp. \\
\hline Rhipicephalus sanguineus & 209 & 70.4 & 9 & 1 & & 5 & 1 \\
\hline $\mathrm{RI}(\%)^{*}$ & & & 0.9 & 0.09 & & 0.5 & 0.09 \\
\hline Rhipicephalus turanicus & 58 & 19.5 & 12 & & 5 & 1 & 2 \\
\hline $\mathrm{RI}(\%)^{\star}$ & & & 4.5 & & 1.8 & 0.3 & 0.7 \\
\hline Rhipicephalus bursa & 18 & 6.1 & & & & & 1 \\
\hline $\mathrm{RI}(\%)^{*}$ & & & & & & & 1.1 \\
\hline Rhipicephalus pusillus & 1 & 0.3 & 1 & & & & \\
\hline $\mathrm{RI}(\%)^{*}$ & & & 100 & & & & \\
\hline Haemaphysalis sulcata & 5 & 1.7 & & 3 & & 1 & \\
\hline $\mathrm{RI}(\%)^{\star}$ & & & & 16.7 & & 4.4 & \\
\hline Dermacentor marginatus & 3 & 1 & & 1 & & & \\
\hline $\mathrm{RI}(\%)^{*}$ & & & & 7.8 & & & \\
\hline
\end{tabular}

${ }^{\star} \mathrm{MLE}=1-(1-Y / X)^{1 / m}$.

pools (five from sheep, five from goats, one from cattle and one from a horse) of Rhipicephalus turanicus were positive for Rickettsia SFG, five pools (one from sheep and four from goats) were positive for A. phagocytophilum, one pool from a goat was positive for $C$. burnetii and two pools (one from a dog and one from a goat) were positive for Bartonella species. One pool of Rhipicephalus bursa from a goat was positive for Bartonella species. The pool of Rhipicephalus pusillus from a hedgehog was positive for Rickettsia SFG. Hyalomma marginatum marginatum ticks were grouped in three pools, which tested negative for all pathogens considered in this study. Haemaphysalis sulcata ticks were grouped in five pools: three pools from goats were positive for $E$. canis, and one was positive for $C$. burnetii. Finally, positivity to E. canis was detected in one out of three D. marginatus pools, from a wild boar. No positivity to Leishmania species was detected. Data regarding the prevalence of tick infection by pathogens are summarized in Table 1.

\section{DISCUSSION}

In this paper, we report the results of a 2-year survey carried out in two areas of south-eastern Sardinia with the aim of evaluating tick distribution and circulation of micro-organisms in ticks collected from different mammals. Rhipicephalus sanguineus was confirmed to be the most represented tick species in Sardinia, accounting for over $70 \%$ of all ticks examined. Rhipicephalus sanguineus, which is considered to be a dog-associated species and was found mostly on these animals also in this study, is well adapted to live in the mesomediterranean bioclimatic zone; as a consequence, it can feed in all stages, and generally does not require other host species to complete its life cycle (Psaroulaki et al., 2006). Rhipicephalus turanicus is considered the species mostly associated with sheep (Genchi \& Manfredi, 1999). This was in agreement with our study; in fact, most of the Rhipicephalus turanicus ticks were removed from small ruminants. Rhipicephalus bursa ticks were also found in this study. In the Mediterranean basin, this species is considered a major ectoparasite of sheep (Yeruham et al., 2000), from which it was also recovered in high percentages in this work. However, although it was retrieved mostly from small ruminants, we found Rhipicephalus bursa in many other host species, which might act as vectors facilitating the spread of this tick species among flocks.

The Rhipicephalus pusillus ticks identified were all collected from a hedgehog; in fact, although these ticks are reported to inhabit rabbits, on which they feed during all stages, they can occasionally infest hedgehogs and rodents (Walker et al., 2000), consistent with our findings. We also recovered Hyalomma marginatum marginatum, only from cattle and not from other host species. Haemaphysalis sulcata was detected only on small ruminants, in accordance with the studies carried out by Genchi \& Manfredi (1999), who reported the frequent occurrence of these species on small ruminants when they are reared on pastures, mainly in central-southern Italy. All ticks identified as D. marginatus were found only in wild boars. These ticks require warm, dry habitats and inhabit wild boars, which constitute the main hosts (Ortuño et al., 2006). Ixodes marginatus was not detected in this study. However, this is not surprising, since it has been previously reported as a tick species scarcely present in Sardinia (Alberti et al., 2005a). 
Table 2. Association between different tick species and the mammals from which they were collected

\begin{tabular}{|c|c|c|c|}
\hline Tick species & Total ticks & Total pools & Mammalian host \\
\hline \multirow[t]{5}{*}{ Rhipicephalus sanguineus } & 965 & 193 & Dog \\
\hline & 45 & 9 & Sheep \\
\hline & 20 & 4 & Goat \\
\hline & 10 & 2 & Cattle \\
\hline & 5 & 1 & Hedgehog \\
\hline Total & 1045 & 209 & \\
\hline \multirow[t]{5}{*}{ Rhipicephalus turanicus } & 5 & 1 & Dog \\
\hline & 95 & 19 & Sheep \\
\hline & 150 & 30 & Goat \\
\hline & 35 & 7 & Cattle \\
\hline & 5 & 1 & Horse \\
\hline Total & 290 & 58 & \\
\hline \multirow[t]{5}{*}{ Rhipicephalus bursa } & 35 & 7 & Sheep \\
\hline & 15 & 3 & Goat \\
\hline & 5 & 1 & Cattle \\
\hline & 30 & 6 & Horse \\
\hline & 5 & 1 & Deer \\
\hline Total & 90 & 18 & \\
\hline Rhipicephalus pusillus & 5 & 1 & Hedgehog \\
\hline Total & 5 & 1 & \\
\hline \multirow[t]{2}{*}{ Haemaphysalis sulcata } & 5 & 1 & Sheep \\
\hline & 20 & 4 & Goat \\
\hline Total & 25 & 5 & \\
\hline $\begin{array}{l}\text { Hyalomma marginatum } \\
\text { marginatum }\end{array}$ & 15 & 3 & Cattle \\
\hline Total & 15 & 3 & \\
\hline Dermacentor marginatus & 15 & 3 & Wild boar \\
\hline Total & 15 & 3 & \\
\hline Overall total & 1485 & 297 & \\
\hline
\end{tabular}

This study provides data regarding the prevalence of Rickettsia species, E. canis, A. phagocytophilum, C. burnetii, Bartonella species and Leishmania species potentially transmitted by ticks in Sardinia. According to speciesspecificity in tick behaviour, Ixodida ticks of the genera Rhipicephalus, Dermacentor, Ixodes and Amblyomma are the most important vectors of Rickettsiae species including human rickettsial pathogens (Duh et al., 2006).

Ticks of the family Ixodidae can transmit Anaplasma species and Ehrlichia species, which are closely related to the genus Rickettsia. Rhipicephalus sanguineus is also the primary vector of E. canis (Murphy et al., 1998); this observation was confirmed in this study. Here, PCR evidence of $E$. canis was detected not only in Rhipicephalus sanguineus but also in Rhipicephalus turanicus, Haemaphysalis sulcata and D. marginatus. Other authors have found PCR evidence of E. canis in pools of Rhipicephalus sanguineus collected from Venezuela (Unver et al., 2001), Albania (Christova et al., 2003) and Oklahoma
(Murphy et al., 1998). The detection of five A. phagocytophilum-positive pools in Rhipicephalus turanicus collected from small ruminants was in accordance with the studies carried out by Keysary et al. (2007). However, we did not find PCR-positivity for A. phagocytophilum in Rhipicephalus sanguineus (Alberti et al., 2005a), D. marginatus or Rhipicephalus bursa (Merino et al., 2005), although this might be due to the sample size or to the different geographical areas where ticks were collected. However, our finding of $A$. phagocytophilum positivity also in other tick species parasitizing different animal hosts to those previously reported is a relevant finding for human and animal health (Alberti et al., 2005b; Ruscio \& Cinco, 2003; Mastrandrea et al., 2006).

In this work, Rhipicephalus sanguineus, Rhipicephalus turanicus and Haemaphysalis sulcata were positive for C. burnetii. This pathogen is reported to be carried by several tick species: Dermacentor species in Germany (Sting et al., 2004), plus Rhipicephalus sanguineus and Hyalomma 
species ticks in Cyprus (Spyridaki et al., 2002). Infection by C. burnetii in Rhipicephalus turanicus was detected in the Greek island of Cephalonia (Psaroulaki et al., 2006).

It is known that Rhipicephalus sanguineus ticks could be potential vectors of Bartonella species, as has been hypothesized since 1992 (Lucey et al., 1992). Experimental vector transmission studies must be performed to validate the hypothesis that ticks transmit Bartonella species to animals and humans (Billeter et al., 2008). In our work, not only Rhipicephalus sanguineus, but also Rhipicephalus turanicus and Rhipicephalus bursa, showed positivity for Bartonella species.

We did not find positivity to Leishmania species in all seven tick species analysed. The vectorial competence of Rhipicephalus sanguineus in relation to the biology of Leishmania and to the epidemiology of canine leishmaniasis is strongly questionable, taking into account the strict association of this tick species with dogs and the low indices of natural leishmanial infection as presented by Coutinho et al. (2005).

These results increase our knowledge of tick-borne diseases in Sardinia, and provide a useful contribution to understanding their epidemiology. These findings might be helpful for evaluating patient health problems and for risk prevention, and could provide the basis for a plan aimed at monitoring the spread of tick-borne diseases in the island.

\section{ACKNOWLEDGEMENTS}

The authors thank Dr Sandro Rolesu for data analysis, and Dr Maria Filippa Addis for her critical reading and suggestions on the manuscript.

\section{REFERENCES}

Alberti, A., Addis, M. F., Sparagano, O., Zobba, R., Chessa, B., Cubeddu, T., Pinna Parpaglia, M. L., Ardu, M. \& Pittau, M. (2005a). Anaplasma phagocytophilum, Sardinia, Italy. Emerg Infect Dis 11, 1322-1324.

Alberti, A., Zobba, R., Chessa, B., Addis, M. F., Sparagano, O., Pinna Parpaglia, M. L., Cubeddu, T., Pintori, G. \& Pittau, M. (2005b). Equine and canine Anaplasma phagocytophilum strains isolated on the island of Sardinia (Italy) are phylogenetically related to pathogenic strains from the United States. Appl Environ Microbiol 71, 6418-6422.

Angelakis, E., Billeter, S. A., Breitschwerdt, E. B., Chomel, B. B. \& Raoult, D. (2010). Potential for tick-borne bartonelloses. Emerg Infect Dis 16, 385-391.

Beninati, T., Lo, N., Noda, H., Esposito, F., Rizzoli, A., Favia, G. \& Genchi, C. (2002). First detection of spotted fever group rickettsiae in Ixodes ricinus from Italy. Emerg Infect Dis 8, 983-986.

Bernasconi, M. V., Casati, S., Péter, O. \& Piffaretti, J. C. (2002). Rhipicephalus ticks infected with Rickettsia and Coxiella in Southern Switzerland (Canton Ticino). Infect Genet Evol 2, 111-120.

Billeter, S. A., Levy, M. G., Chomel, B. B. \& Breitschwerdt, E. B. (2008). Vector transmission of Bartonella species with emphasis on the potential for tick transmission. Med Vet Entomol 22, 1-15.
Black, W. C. \& Piesman, J. (1994). Phylogeny of hard- and soft-tick taxa (Acari: Ixodida) based on mitochondrial $16 \mathrm{~S}$ rDNA sequences. Proc Natl Acad Sci U S A 91, 10034-10038.

Christova, I., Van De Pol, J., Yazar, S., Velo, E. \& Schouls, L. (2003). Identification of Borrelia burgdorferi sensu lato, Anaplasma and Ehrlichia species, and spotted fever group Rickettsiae in ticks from Southeastern Europe. Eur J Clin Microbiol Infect Dis 22, 535-542.

Cotté, V., Bonnet, S., Le Rhun, D., Le Naour, E., Chauvin, A., Boulouis, H. J., Lecuelle, B., Lilin, T. \& Vayssier-Taussat, M. (2008). Transmission of Bartonella henselae by Ixodes ricinus. Emerg Infect Dis 14, 1074-1080.

Coutinho, M. T., Lacerda, L., Sterzik, A., Fujiwara, R. T., Botelho, J. R., De Maria, M., Genaro, O. \& Linardi, P. M. (2005). Participation of Rhipicephalus sanguineus (Acari: Ixodidae) in the epidemiology of canine visceral leishmaniasis. Vet Parasitol 128, 149-155.

Dantas-Torres, F. (2008). The brown dog tick, Rhipicephalus sanguineus (Latreille, 1806) (Acari: Ixodidae): from taxonomy to control. Vet Parasitol 152, 173-185.

Duh, D., Punda-Polic, V., Trilar, T., Petrovec, M., Bradarić, N. \& AvsicZupanc, T. (2006). Molecular identification of Rickettsia felis-like bacteria in Haemaphysalis sulcata ticks collected from domestic animals in southern Croatia. Ann N Y Acad Sci 1078, 347-351.

Foley, J. E., Nieto, N. C., Adjemian, J., Dabritz, H. \& Brown, R. N. (2008). Anaplasma phagocytophilum infection in small mammal hosts of Ixodes ticks, western United States. Emerg Infect Dis 14, 1147-1150.

Genchi, C. \& Manfredi, M. T. (1999). Tick species infesting ruminants in Italy: ecological and bio-climatic factors affecting the different regional distribution. Parassitologia 41, 41-45.

Keysary, A., Massung, R. F., Inbar, M., Wallach, A. D., Shanas, U., Mumcuoglu, K. Y. \& Waner, T. (2007). Molecular evidence for Anaplasma phagocytophilum in Israel. Emerg Infect Dis 13, 14111412.

Kolbert, C. (1996). Detection of the agent of human granulocytic ehrlichiosis by PCR. In PCR Protocols for Emerging Infectious Diseases, pp. 106-111. Edited by D. H. Persing. Washington, DC: American Society for Microbiology.

Lucey, D., Dolan, M. J., Moss, C. W. \& Garcia, M. (1992). Relapsing illness due to Rochalimaea henselae in immunocompetent hosts: implication for therapy and new epidemiological associations. Clin Infect Dis 14, 683-688.

Manilla, G. (1998). Acari Ixodidae, Fauna d'Italia. Bologna: Edizioni Calderini.

Mastrandrea, S., Mura, M. S., Tola, S., Patta, C., Tanda, A., Porcu, R. \& Masala, G. (2006). Two cases of human granulocytic ehrlichiosis in Sardinia, Italy, confirmed by PCR. Ann N Y Acad Sci 1078, 548551.

Merino, F. J., Nebreda, T., Serrano, J. L., Fernández-Soto, P., Encinas, A. \& Pérez-Sánchez, R. (2005). Tick species and tick-borne infections identified in population from a rural area of Spain. Epidemiol Infect 133, 943-949.

Murphy, G. L., Ewing, S. A., Whitworth, L. C., Fox, J. C. \& Kocan, A. A. (1998). A molecular and serologic survey of Ehrlichia canis, E. chaffeensis, and E. ewingii in dogs and ticks from Oklahoma. Vet Parasitol 79, 325339.

Noda, H., Munderloh, U. G. \& Kurtti, T. J. (1997). Endosymbionts of ticks and their relationship to Wolbachia spp. and tick-borne pathogens of humans and animals. Appl Environ Microbiol 63, 3926-3932.

Ortuño, A., Quesada, M., López, S., Miret, J., Cardeñosa, N., Castellà, N., Anton, E. \& Segura, F. (2006). Prevalence of Rickettsia slovaca in Dermacentor marginatus ticks removed from wild boar (Sus scrofa) in northeastern Spain. Ann N Y Acad Sci 1078, 324-327. 
Papadopoulos, B., Morel, P. C. \& Aeschlimann, A. (1996). Ticks of domestic animals in the Macedonia region of Greece. Vet Parasitol 63, 25-40.

Parola, P. \& Raoult, D. (2001). Ticks and tick-borne bacterial diseases in humans: an emerging infectious threat. Clin Infect Dis 32, 897928.

Psaroulaki, A., Ragiadakou, D., Kouris, G., Papadopoulos, B., Chaniotis, B. \& Tselentis, Y. (2006). Ticks, tick-borne rickettsiae, and Coxiella burnetii in the Greek island of Cephalonia. Ann N Y Acad Sci 1078, 389-399.

Ruscio, M. \& Cinco, M. (2003). Human granulocytic ehrlichiosis in Italy. First report on two confirmed cases. Ann N Y Acad Sci 990, 350352.

Sander, A., Posselt, M., Böhm, N., Ruess, M. \& Altwegg, M. (1999). Detection of Bartonella henselae DNA by two different PCR assays and determination of the genotypes of strains involved in histologically defined cat scratch disease. J Clin Microbiol 37, 993-997.

Sarih, M., M'Ghirbi, Y., Bouattour, A., Gern, L., Baranton, G. \& Postic, D. (2005). Detection and identification of Ehrlichia spp. in ticks collected in Tunisia and Morocco. J Clin Microbiol 43, 11271132.

Spyridaki, I., Psaroulaki, A., Loukaides, F. \& Antoniou, M. (2002). Isolation of Coxiella burnetii by a centrifugation shell vial assay from ticks collected in Cyprus: detection by nested polymerase chain reaction (PCR) and by PCR-restriction fragment length polymorphism analyses. Am J Trop Med Hyg 66, 86-90.
Stein, A. \& Raoult, D. (1992). Detection of Coxiella burnetii by DNA amplification using polymerase chain reaction. J Clin Microbiol 30, 2462-2466.

Stich, R. W., Rikihisa, Y., Ewing, S. A., Needham, G. R., Grover, D. L. \& Jittapalapong, S. (2002). Detection of Ehrlichia canis in canine carrier blood and in individual experimentally infected ticks with a $p 30$-based PCR assay. J Clin Microbiol 40, 540-546.

Sting, R., Breitling, N., Oehme, R. \& Kimmig, P. (2004). The occurrence of Coxiella burnetii in sheep and ticks of the genus Dermacentor in Baden-Wuerttemberg. Dtsch Tierarztl Wochenschr 111, 390-394.

Unver, A., Perez, M., Orellana, N., Huang, H. \& Rikihisa, Y. (2001). Molecular and antigenic comparison of Ehrlichia canis isolates from dogs, ticks, and a human in Venezuela. J Clin Microbiol 39, 2788-2793.

van Eys, G. J., Schoone, G. J., Kroon, N. C. \& Ebeling, S. B. (1992). Sequence analysis of small subunit ribosomal RNA genes and its use for detection and identification of Leishmania parasites. Mol Biochem Parasitol 51, 133-142.

Walker, J. B., Keirans, J. E. \& Horak, I. G. (2000). The Genus Rhipicephalus (Acari, Ixodidae). A Guide to the Brown Ticks of the World. Cambridge: Cambridge University Press.

Walter, S. D., Hildreth, S. W. \& Beaty, B. J. (1980). Estimation of infection rates in populations of organisms using pools of variable size. Am J Epidemiol 112, 124-128.

Yeruham, I., Hadani, A. \& Galker, F. (2000). The life cycle of Rhipicephalus bursa Canestrini and Fanzago, 1877 (Acarina: ixodidae) under laboratory conditions. Vet Parasitol 89, 109-116. 